\title{
Lactobacillus acidophilus Restores Functionality in Uremic Macrophages: Plausible or Lacking Evidence?
}

\author{
Luis Vitetta ${ }^{1,2}$
}

Published online: 18 February 2016

(c) Springer Science+Business Media New York 2016

Over the past several decades, research has redefined the interactions between gut microbes and vertebrates, now recognizing that the intestinal microbiome and its mammalian host have shared co-evolutionary metabolic interactions that span millennia. Studies from the Human Microbiome Project (HMP) have estimated that humans possess ten times more bacterial cells than there are human cells. In the gastrointestinal (GI) tract, the bacterial cohort contributes approximately 3.3 million non-redundant microbial genes, far exceeding that of the human input [1]. This genetic diversity is likely to provide the necessary cues through host-microbial interactions for the development of regulated signals that promote immunological tolerance, metabolic regulation, and stability, and other factors that may then help control local and extra-intestinal end-organ (e.g., renal, hepatic) physiology.

The intestinal mucosa is the largest and most dynamic immunological environment of the body. Often the first point of pathogen or antigen exposure, many microbes use it as a point of entry into the rest of the body. The gut immune system therefore needs to be prepared to respond to insults either from pathogens or other molecular triggers while at the same time not responding to other presumably innocuous environmental antigens, food particles, and commensal bacteria and their respective metabolites. Misdirected immune responses to antigens thought harmless are thought by some to be the underlying cause of food allergies and debilitating conditions such as inflammatory

Luis Vitetta

luis.vitetta@sydney.edu.au; luis_vitetta@medlab.co

1 Sydney Medical School, The University of Sydney, Sydney, NSW, Australia

2 Medlab Clinical, Sydney, NSW, Australia bowel diseases with their unregulated and overcompensated, pro-inflammatory sequelae [2]. As a consequence, dysbiosis of the GI tract describes alteration of bacterial populations thought to increase the risk of developing gut barrier dysfunction with consequent increased permeability, also referred to as a "leaky gut," with consequent translocation of bacteria, bacterial endotoxins, or environmental antigens across the gut wall. While the immune system is thought to be involved, mechanistic data that link immune function disruption to adverse end-organ physiological functions, which are present for the kidney, are mostly missing for the gut.

The supposition that the GI tract bacterial cohort could indirectly and adversely influence the physiological function of an end-organ such as the liver or kidney, by contributing pro-inflammatory activity in the gut mucosa, is a novel concept with biological plausibility [2,3]. Uremic toxins (e.g., glycation end products, $p$-cresyl sulfate, indoxyl sulfate) can produce pro-inflammatory responses, including leukocyte stimulation and endothelial dysfunction, which can promote GI tract dysbiosis [3-5]. Therefore, it is highly probable that the 100 trillion bacteria in the GI tract could negatively and positively influence the nutritive, metabolic, physiological, and importantly immunological functions of the host [2]. The diversity of commensal bacteria in the GI tract can, in turn, account for the varied macrophage responses that have been encountered in the intestines [6].

Macrophages, in their eradication of pathogenic microbes so as to maintain homeostasis, are an essential component of innate immunity. The activated phenotype exists in macrophages that present antigens to $\mathrm{T}$ lymphocytes in order to initiate an appropriate immune response after recognition of microbial proteins [7]. In addition to serving as antigen-presenting cells, activated macrophages 
secrete a range of cytokines such as interleukin (IL)-1, interferon (IFN)- $\alpha$, and cytotoxic proteins that activate $\mathrm{T}$ cell lymphocytes [7]. Moreover as part of their overall action, macrophages can phagocytize exogenous antigens, cellular debris, insoluble particles, and activated clotting factors [7].

In this issue of Digestive Diseases and Sciences, Sun and colleagues [8] gavaged the probiotic Lactobacillus acidophilus (strain LB) to male Sprague-Dawley rats with induced uremia. They reported that in the uremic rats, the green fluorescent protein-labeled tracer bacteria were observed in the ileum, in mononuclear leukocytes, and in the liver and spleen. Since the macrophage marker CD68 fluoresced red in the same organs, the bacteria were considered to be localized to the macrophages. Whereas the green fluorescent signal for bacteria and the red fluorescence signal for the macrophage marker were rare in the ileum of the sham group of animals, in the uremic rat group gavaged with the Lactobacillus acidophilus LB, colocalization was present in the ileum.

Compared to controls, macrophages from the uremic animals had a less activated phenotype, with fewer cytoplasmic protrusions and pseudopodia. In the study by Sun and colleagues [8], uremia was associated with depressed macrophage phagocytic activity associated with disrupted macrophage signaling, migration, and chemotaxis when compared to normal activated macrophages that characteristically have greater phagocytic activity than do resting cells [7]. The macrophages were loaded with bacteria, suggesting that bacteria translocated across the intestinal epithelial barrier into the intestinal mucosa, a challenge that would then induce a pro-inflammatory response.

The probiotic bacterium Lactobacillus acidophilus strain LB was associated with reduced bacterial translocation in the uremic rats and enhanced macrophage phagocytosis, indicative of recovered macrophage defense capability. This strain is not only antibacterial [9], but also reduces epithelial barrier lesions induced by pathogenic bacteria such as the diarrheagenic and diffusely adhering Escherichia coli strain C1845 [10].

The gut physical barrier, formed by intestinal epithelial cells, maintains homeostasis in the intestine in a continuous cooperative process with the innate immune system, importantly linking it to intestine-resident macrophages and to dendritic cells [11]. Intestine-resident macrophages $\left(\mathrm{CX}_{3} \mathrm{CR} 1^{\mathrm{hi}}\right)$ that are derived from blood monocytes $\left(\right.$ Ly6C $\left.C^{+}\right)$persist in close physical proximity with intestinal epithelial cells. The close physical contact of the macrophages provides a dedicated phagocytic function that clears pathogens and commensal bacteria that otherwise can traverse the mucosal barrier. Furthermore, macrophage expression of tight junction proteins enables the formation of transepithelial dendrites that penetrate into the lumen of the intestine for continuous sampling of luminal exogenous antigens $[11,12]$. Moreover, the $\mathrm{CX}_{3} \mathrm{CR} 1^{\mathrm{hi}}$ macrophage populations maintain mucosal tolerance by improving the survival of and locally expanding previously primed $\mathrm{T}$ lymphocytes [11]. In concert with this activity, $\mathrm{CX}_{3} \mathrm{CR} 1^{\mathrm{hi}}$ macrophages promote tolerance in the intestinal lamina propria by elaborating the anti-inflammatory cytokine IL10, which together suppress pro-inflammatory cytokine production by colitogenic $\mathrm{T}$ lymphocytes while promoting regulatory $\mathrm{T}$ lymphocyte function [11]. As a consequence, components of the local innate immune system (i.e., macrophages and dendritic cells) and the intestinal epithelia interact to maintain a tolerogenic functional state. The intestinal epithelium produces soluble protein factors (e.g., thymic stromal lymphopoietin, transforming growth factor- $\beta$, and retinoic acid) and also expresses the integrin ligand semaphorin $7 \mathrm{~A}$ that undergoes contact-dependent interactions with intestinal macrophages, inducing expression of IL-10 that in turn promotes intestinal homeostasis.

The purported health effects accruing from the consumption of lactic acid bacteria has a centuries-long history. Probiotic bacteria like those from the Lactobacilli and Bifidobacteria genera as well as others are not only essential to the production of fermented dairy products such as yogurts, but are also consumed directly as dietary supplements with possible health benefits [13]. In mostly experimental studies, probiotic bacteria affect gut immune function, dependent upon the strain, setting, immunological functions measured, and the type of cells that are acted upon [14].

At present, translational research is needed to test the hypothesis that the administration of a single probiotic strain to humans may ameliorate inflammatory conditions by reducing macrophage-assisted bacterial translocation. Since the administration of probiotics to humans is generally considered as safe [15], the bar is set low for the conduct of well-designed, randomized controlled trials testing for strain-specific effects.

\section{Compliance with ethical standards}

Conflict of interest Luis Vitetta has received National Institute of Complementary Medicine and National Health and Medical Research Council of Australia competitive funding and Industry support for research into probiotics and the gut microbiome. The author has no further conflicts of interest relevant to the content of this editorial.

\section{References}

1. Qin J, Li R, Raes J, et al. A human gut microbial gene catalogue established by metagenomic sequencing. Nature. 2010;464:59-65.

2. Vitetta L, Linnane AW, Gobe GC. From the gastrointestinal tract (GIT) to the kidneys: live bacterial cultures (probiotics) mediating reductions of uremic toxin levels via free radical signaling. Toxins. 2013;5:2042-2057. 
3. Ramezani A, Raj DS. The gut microbiome, kidney disease, and targeted interventions. J Am Soc Nephrol (JASN). 2014;25: 657-670.

4. Vitetta L, Gobe G. Uremia and chronic kidney disease: the role of the gut microflora and therapies with pro- and prebiotics. Mol Nutr Food Res. 2013;57:824-832.

5. Vanholder R, Glorieux G. The intestine and the kidneys: a bad marriage can be hazardous. Clin Kidney J. 2015;8:168-179.

6. Ivanov II, Honda K. Intestinal commensal microbes as immune modulators. Cell Host Microbe. 2012;12:496-508.

7. Sansonetti P. Host-pathogen interactions: the seduction of molecular cross talk. Gut.. 2002;50:Iii2-Iii8.

8. Sun $\mathrm{L}$, Liu $\mathrm{H}$, Jiang $\mathrm{H}$, et al. Macrophages are involved in gut bacterial translocation and reversed by lactobacillus in experimental uremia. Dig Dis Sci. (Epub ahead of print). doi:10.1007/ s10620-015-3950-z.

9. Lievin-Le Moal V, Servin AL. Anti-infective activities of lactobacillus strains in the human intestinal microbiota: from probiotics to gastrointestinal anti-infectious biotherapeutic agents. Clin Microbiol Rev. 2014;27:167-199.
10. Lievin-Le Moal V, Amsellem R, Servin AL, Coconnier MH. Lactobacillus acidophilus (strain LB) from the resident adult human gastrointestinal microflora exerts activity against brush border damage promoted by a diarrhoeagenic Escherichia coli in human enterocyte-like cells. Gut. 2002;50:803-811.

11. Peterson LW, Artis D. Intestinal epithelial cells: regulators of barrier function and immune homeostasis. Nat Rev Immunol. 2014;14:141-153.

12. Niess JH, Brand S, Gu X, et al. CX3CR1-mediated dendritic cell access to the intestinal lumen and bacterial clearance. Science (New York, NY). 2005;307:254-258.

13. Morelli L, Capurso L. FAO/WHO guidelines on probiotics: 10 years later. J Clin Gastroenterol. 2012;46:S1-S2.

14. Delcenserie V, Martel D, Lamoureux M, Amiot J, Boutin Y, Roy D. Immunomodulatory effects of probiotics in the intestinal tract. Curr Issues Mol Biol. 2008;10:37-54.

15. Snydman DR. The safety of probiotics. Clin Infect Dis. 2008;46:S104-S111. (discussion S44-S51). 